\title{
On the boundary element formulation to compute critical loads considering the effect of shear deformation in plate bending
}

\author{
L. Palermo Jr. \& R. A. Soares Jr. \\ School of Civil Engineering, Architecture and Urban Design, \\ State University of Campinas, Brazil
}

\begin{abstract}
A boundary element formulation to compute critical loads in static problems is analyzed. The bending model includes the effect of shear deformation that turns deflection derivatives independent of plate rotations. The effect of geometrical non-linearity (GNL) related to in-plane loading is introduced with two additional integrals in the formulation: one is performed on the domain and the other on the boundary. The boundary integral can be related to one of the natural conditions according to the boundary value problem. The inverse iteration was used to get the lowest eigenvalue. Results obtained with several boundary conditions and using derivatives of the deflection in the effect of GNL are compared and discussed with expect values from the literature.
\end{abstract}

Keywords: plate bending with in-plane forces, critical loads of plates, ReissnerMindlin plates.

\section{Introduction}

A formulation to compute critical loads in plates is studied with the boundary element method (BEM). The effect of shear deformation is included in the bending model due to its benefits on plate behavior modeling as shown by Reissner on the assessment of stress concentration around holes [1] and by Mindlin on the wave propagation analysis considering short wavelengths [2]. The effect of the geometrical of non-linearity (GNL) related to in-plane loading is introduced according to the development in [3]. In spite of the fact that classical plate theory has been used in [3], the formulation for the effect of GNL is considered in bending models including the effect of shear deformation when 
thin or thin to thick plate types are analyzed (as shown in several studies in the literature).

A review on the literature until 1989 on the buckling of thin rectangular plates was done by Jones in [4]. Dawe and Roufaeil [5] discussed the effect of GNL in plate bending considering the effect of shear deformation since the first study presented by Herrmann and Armenakas [6]. Doong [7] and Matsunaga [8] studied vibration and buckling of thick plates employing a high order deformation theory for the bending model. The buckling analyses of skew plates considering the Mindlin model was done by Kitipornchai et al. [9] with pb-2 Rayleigh-Ritz method. Lei et al. [10] formulated an integral equation for buckling analysis of Reissner plates, where the domain integral related to GNL was discretized using constant triangular cells. A similar study was done by Purbolaksono and Aliabadi [11] but using constant rectangular cells.

A boundary element formulation using first derivatives of the deflection to introduce the effect of GNL in bending of plates was presented in $[12,13]$. The elastodynamic solution [14] was used and critical loads were computed according to the frequency value. It is well known that the frequency value modifies the value of critical in-plane force and vice-versa [15]. The values obtained for critical loads at near zero frequencies were close to expected values from the literature for the static condition. A question appeared: Was the numerical implementation or the near zero value for the frequency that improved the formulation to compute critical loads at "the static condition"?

An analysis of the formulation using first derivatives of the deflection in the effect of GNL is done in this study. The static fundamental solution [16] is used to remove the dynamic effect whereas other features in $[12,13]$ remained. The effect of geometrical non-linearity (GNL) is introduced with two additional integrals in the formulation: one is performed on the domain and the other on the boundary. The boundary integral can be related to one of the natural conditions according to the boundary value problem. Isoparametric quadratic boundary elements and constant rectangular domain cells are employed. The inverse iteration and Rayleigh quotient are used to get the lowest eigenvalue. Results obtained with several boundary conditions are compared and discussed with expect values from the literature.

\section{Boundary integral equations}

The constitutive equations for an isotropic and homogeneous plate material are:

$$
\begin{gathered}
M_{\alpha \beta}=D \frac{(1-v)}{2}\left(\psi_{\alpha, \beta}+\psi_{\beta, \alpha}+\frac{2 v}{1-v} \psi_{\gamma, \gamma} \delta_{\mathrm{a} \beta}\right)+\delta_{\alpha \beta} q R E \\
Q_{\alpha}=D \frac{(1-v)}{2} \lambda^{2}\left(\psi_{\alpha}+w_{, \alpha}\right)
\end{gathered}
$$

with

$$
\lambda^{2}=12 \frac{\kappa^{2}}{h^{2}} ; \quad R E=\frac{v}{\lambda^{2}(1-v)}
$$


The plate has a uniform thickness $\mathrm{h}, \mathrm{D}$ is the flexural rigidity, $v$ is the Poisson ratio, $\mathrm{w}$ is the deflection, $\psi_{\alpha}$ is the plate rotation in the direction $\alpha$ and $\delta_{\alpha \beta}$ is the Kronecker delta. The product qRE in equation (1) corresponds to the linearly weighted average effect of the normal stress component in the thickness direction and should be considered in Reissner's model [1] but not in Mindlin's model [2], in which it should be considered to be null. The shear parameter $\kappa^{2}$ is equal to $5 / 6$ and $\pi^{2} / 12$ for the Reissner and Mindlin models, respectively.

The constitutive eqns (1) and (2) employed a unified notation for the Reissner and the Mindlin model and the convention of this study was used, i.e. Latin indices take values $\{1,2$ and 3$\}$ and Greek indices take values $\{1,2\}$. To match the constitutive equations used in the literature for other numerical techniques [8, 9], the linearly weighted average effect of the normal stress component in the thickness direction (qRE) is assumed null in this study and the difference between Reissner and Mindlin models will be done by the shear parameter.

The natural conditions and the equilibrium equations for the problem can be obtained with the calculus of variations $[17,18]$. The energy functional of the plate is given by:

$$
\begin{aligned}
\Pi= & \int_{\Omega}\left\{\frac{D(1-v)}{4}\left[\psi_{\alpha, \beta}{ }^{2}+\psi_{\alpha, \beta} \psi_{\beta, \alpha}+\frac{2 v}{(1-v)} \psi_{\gamma, \gamma}{ }^{2}+\lambda^{2}\left(\psi_{\alpha}+w_{, \alpha}\right)^{2}\right]\right\} d \Omega+ \\
& \ldots-\int_{\Omega} q w d \Omega-\int_{\Gamma_{f}}\left(P w+E M_{\alpha} \psi_{\alpha}\right) d \Gamma+\int_{\Omega} \frac{1}{2}\left(N_{\alpha \beta} w_{, \alpha} w_{, \beta}\right) d \Omega
\end{aligned}
$$

The energy functional of the plate was written in the complete form in eqn (3) and is similar to that presented in [9]. The first integral (domain integral) is the strain energy whereas the effect of GNL appeared in the last integral. The second and the third integral are the potential energy of the external loads distributed on the domain and on a portion of the boundary line $\left(\Gamma_{\mathrm{f}}\right)$. The out of plane loads on are $\mathrm{q}$ and $\mathrm{P}$ whereas $\mathrm{EM}_{1}, \mathrm{EM}_{2}$ are couples in directions 1,2 , respectively. The displacements $\mathrm{w}, \psi_{1}, \psi_{2}$ are not prescribed on the portion of the boundary line $\Gamma_{\mathrm{f}}$. The energy functional of the plate can be written in a general function to be minimized with the calculus of variations:

$$
\Pi=\int_{\Omega} F\left(w, \psi_{1}, \psi_{2}, w_{, 1}, \psi_{1,1}, \psi_{2,1}, w_{, 2}, \psi_{1,2}, \psi_{2,2}\right) d \Omega
$$

The Euler equations obtained from the minimization of eqn (4) is given by:

$$
\begin{gathered}
\frac{\partial F}{\partial \psi_{\alpha}}-\frac{\partial}{\partial X_{\beta}}\left(\frac{\partial F}{\partial \psi_{\alpha, \beta}}\right)=0 \\
\frac{\partial F}{\partial w}-\frac{\partial}{\partial X_{\beta}}\left(\frac{\partial F}{\partial w_{, \beta}}\right)=0
\end{gathered}
$$


The equilibrium equations are obtained when the constitutive eqns (1) and (2) are introduced in resultant expressions from the application of Euler equations:

$$
\begin{gathered}
M_{\alpha \beta, \beta}-Q_{\alpha}=0 \\
Q_{\alpha, \alpha}+q+\frac{\partial}{\partial x_{\alpha}}\left(N_{\alpha \beta} \frac{\partial w}{\partial x_{\beta}}\right)=0
\end{gathered}
$$

The natural conditions introduce requirements on the boundary portion $\left(\Gamma_{f}\right)$ with not prescribed displacements where the variations of displacements are not null $\left(\delta \mathrm{w} \neq 0, \delta \psi_{\alpha} \neq 0\right)$ :

$$
\begin{gathered}
\left(\frac{\partial F}{\partial w_{, \alpha}} n_{\alpha}\right) \delta w=0 \stackrel{\text { yields }}{\longrightarrow}\left(\frac{\partial F}{\partial w_{, \alpha}} n_{\alpha}\right)=0 \\
t_{3}=P-n_{\alpha} N_{\alpha \beta} w_{, \beta} \\
\left(\frac{\partial F}{\partial \psi_{\alpha, \beta}} n_{\beta}\right) \delta \psi_{\alpha}=0 \stackrel{\text { yields }}{\longrightarrow}\left(\frac{\partial F}{\partial \psi_{\alpha, \beta}} n_{\beta}\right)=0 \\
M_{\alpha}=E M_{\alpha}
\end{gathered}
$$

Equations (1) and (2) were used to obtain $t_{\alpha}\left(t_{\alpha}=M_{\alpha \beta} \cdot n_{\beta}\right)$ and $t_{3}\left(t_{3}=Q_{\alpha} \cdot n_{\alpha}\right)$, respectively, in natural conditions.

The general form of displacement boundary integral equations (DBIEs) with an additional domain integral containing the effect of GNL is next written with the notation proposed by Weeën:

$$
\begin{gathered}
\frac{1}{2} C_{i j}\left(x^{\prime}\right) u_{j}\left(x^{\prime}\right)+\int_{\Gamma}\left[T_{i j}\left(x^{\prime}, x\right) u_{j}(x)-U_{i j}\left(x^{\prime}, x\right) t_{j}(x)\right] d \Gamma(x) \\
=\iint_{\Omega} U_{i 3}\left(x^{\prime}, X\right)\left[q+\frac{\partial}{\partial X_{\alpha}}\left(N_{\alpha \beta} \frac{\partial u_{3}}{\partial X_{\beta}}\right)\right] d \Omega(X)
\end{gathered}
$$

$\mathrm{C}_{\mathrm{ij}}$ is an element of the matrix $\mathrm{C}$ related to the boundary at the source point, which becomes the identity matrix when a smooth boundary is considered. $\mathrm{u}_{\alpha}$ is $\psi_{\alpha}, \mathrm{u}_{3}$ is $\mathrm{w} . \mathrm{U}_{\mathrm{ij}}$ represents the rotation $(\mathrm{j}=1,2)$ or the deflection $(\mathrm{j}=3)$ due to a unit couple $(i=1,2)$ or a unit point force $(i=3)$, respectively, $T_{i j}$ represents the moment $(j=1,2)$ or the shear $(j=3)$ due to a unit couple $(i=1,2)$ or a unit point force $(i=3)$, respectively.

It is well known in plate analyses that the natural condition is introduced for each generalized force $t_{i}$ corresponding to the displacement not prescribed. According to eqn (7) the effect of GNL should be introduced as "an out-plane force" when the deflection is not prescribed (or, free) on the boundary portion [3, 19].

The term related to the effect of GNL in eqns (6) or (9) can be simplified with the equilibrium equations for in-plane forces $\left(\mathrm{N}_{\alpha \beta, \alpha}=0\right)$. The second derivatives of the deflection appear as result from the simplification, as shown in several studies in the literature. The equilibrium equations for in-plane forces were not 
used here but an algebraic manipulation with the divergence theorem was done in the domain integral related to GNL in eqn. (6), i.e.:

$$
\begin{gathered}
\iint_{\Omega} U_{i 3}\left(x^{\prime}, X\right)\left[\frac{\partial}{\partial X_{\alpha}}\left(N_{\alpha \beta} \frac{\partial u_{3}(X)}{\partial x_{\beta}}\right)\right] d \Omega(X)= \\
\int_{\Gamma} n_{\alpha}(x) N_{\alpha \beta}(x) u_{3, \beta}(x) U_{i 3}\left(x^{\prime}, x\right) d \Gamma(x)-\iint_{\Omega} N_{\alpha \beta}(X) u_{3, \beta}(X) U_{i 3, \alpha}\left(x^{\prime}, X\right) d \Omega(X)
\end{gathered}
$$

The algebraic manipulation carried to use first derivatives of the deflection, only, when the effect of GNL is considered. The final DBIE is given by:

$$
\begin{gathered}
\frac{1}{2} C_{i j}\left(x^{\prime}\right) u_{j}\left(x^{\prime}\right)+\int_{\Gamma}\left[T_{i j}\left(x^{\prime}, x\right) u_{j}(x)-U_{i j}\left(x^{\prime}, x\right) t_{j}(x)\right] d \Gamma(x) \\
=\iint_{\Omega} q(X) U_{i 3}\left(x^{\prime}, X\right) d \Omega(X)+\int_{\Gamma} n_{\alpha}(x) N_{\alpha \beta}(x) u_{3, \beta}(x) U_{i 3}\left(x^{\prime}, x\right) d \Gamma(x)+ \\
\quad-\iint_{\Omega} N_{\alpha \beta}(X) u_{3, \beta}(X) U_{i 3, \alpha}\left(x^{\prime}, X\right) d \Omega(X)
\end{gathered}
$$

The boundary integral containing the effect of GNL can be related to the natural condition given by eqn (7) when the boundary portion has the deflection ( $\mathrm{w}$ or $\mathrm{u}_{3}$ ) not prescribed. It can be shown by assuming the boundary $\Gamma$ split into two portions: $\Gamma_{\mathrm{p}}$ and $\Gamma_{\mathrm{f}}$ where displacements are known (prescribed) and unknown (not prescribed or free), respectively.

$$
\begin{gathered}
\frac{1}{2} C_{i j}\left(x^{\prime}\right) u_{j}\left(x^{\prime}\right)+\int_{\Gamma_{f}} T_{i j}\left(x^{\prime}, x\right) u_{j}(x) d \Gamma(x)-\int_{\Gamma_{p}} U_{i j}\left(x^{\prime}, x\right) t_{j}(x) d \Gamma(x)=\cdots \\
\ldots=\int_{\Gamma_{f}} U_{i \alpha}\left(x^{\prime}, x\right) E M_{\alpha}(x) d \Gamma(x)+\int_{\Gamma_{f}} U_{i 3}\left(x^{\prime}, x\right) P(x) d \Gamma(x)+\cdots \\
\ldots-\int_{p} T_{i j}\left(x^{\prime}, x\right) u_{j}(x) d \Gamma(x)+\iint_{\Omega} q(X) U_{i 3}\left(x^{\prime}, X\right) d \Omega(X)+\cdots \\
\ldots+\int_{\Gamma_{p}} n_{\alpha}(x) N_{\alpha \beta}(x) u_{3, \beta}(x) U_{i 3}\left(x^{\prime}, x\right) d \Gamma(x)+\cdots \\
\ldots-\iint_{\Omega} N_{\alpha \beta}(X) u_{3, \beta}(X) U_{i 3, \alpha}\left(x^{\prime}, X\right) d \Omega(X)
\end{gathered}
$$

The left hand side of eqn (11) contains the unknowns i.e. displacements on $\Gamma_{f}$ and forces on $\Gamma_{\mathrm{p}}$. The external loads on the boundary portion $\Gamma_{\mathrm{f}}$ were introduced in the right hand side according to natural conditions shown in eqns (7) and (8). A simplification can be done on the boundary portion $\Gamma_{\mathrm{f}}$ due to opposite signals in the natural condition and in the boundary integral with the effect with GNL. The boundary integral with the effect of GNL computed only on the boundary portion with prescribed displacements $\left(\Gamma_{\mathrm{p}}\right)$ is the result from the simplification.

The BIE for the first derivative of the deflection in the direction $\gamma$ at an internal point is obtained by differentiating the DBIE given by eqn (10) with respect to the coordinate of the source point ( $\left.\mathrm{X}^{\prime}\right)$. The result is next written in terms of differentiation of coordinates of the field point and with direction 
cosines of the outward normal at the field point written off the differential operator [20]:

$$
\begin{gathered}
u_{3, \gamma}\left(X^{\prime}\right)=\int_{\Gamma}\left\{n_{\alpha}(x) \frac{\partial}{\partial x_{\gamma}}\left[M_{3 \alpha \beta}\left(X^{\prime}, x\right)\right] u_{\beta}(x)+\cdots\right. \\
\ldots+n_{\beta}(x) \frac{\partial}{\partial x_{\gamma}}\left[Q_{3 \beta}\left(X^{\prime}, x\right)\right] u_{3}(x)-\frac{\partial}{\partial x_{\gamma}}\left[U_{3 \beta}\left(X^{\prime}, x\right)\right] t_{\beta}(x)+\cdots \\
\left.\ldots-\frac{\partial}{\partial x_{\gamma}}\left[U_{33}\left(X^{\prime}, x\right)\right] t_{3}(x)\right\} d \Gamma(x)-\iint_{\Omega} \frac{\partial}{\partial X_{\gamma}}\left[U_{33}\left(X^{\prime}, X\right)\right] q(X) d \Omega(X) \ldots \\
\ldots-\int_{\Gamma} n_{\alpha}(x) N_{\alpha \beta}(x) u_{3, \beta}(x) \frac{\partial}{\partial x_{\gamma}}\left[U_{i 3}\left(X^{\prime}, x\right)\right] d \Gamma(x)+\ldots \\
\ldots+\iint_{\Omega} N_{\alpha \beta}(X) u_{3, \beta}(X) \frac{\partial}{\partial X_{\gamma}}\left[U_{i 3, \alpha}\left(X^{\prime}, X\right)\right] d \Omega(X)
\end{gathered}
$$

\section{Numerical implementation}

There were employed quadratic shape functions for isoparametric boundary elements with collocation points always placed on the boundary. The same mapping function was used for conformal and non-conformal interpolations, i.e. nodes at ends of quadratic elements remain at ends when discontinuous elements were employed. The collocation points were placed at nodes in case of continuous elements and at positions $(-0.67,0.0,+0.67)$, in the range $(-1,1)$, in case of discontinuous elements, i.e. the collocation points were shifted to inside the element at the corresponding end where the discontinuity exists. The singularity subtraction [21] and the transformation of variable technique [22] were employed for the Cauchy and the weak type singularity, respectively, when integrations were performed on elements containing the collocation points. The standard Gauss-Legendre scheme was employed for integrations on elements (or, side of the cell) not containing the collocation points. Rectangular cells were used to discretize the domain integral related to the geometrical non-linearity effect. The derivatives of the deflection at the center of the cell were assumed constant on the cell. This assumption allowed to use the divergence theorem to convert the domain integral in equivalent boundary integral performed on sides of the cell. This strategy carried to a simplification on the use of integrals containing the effect of GNL because they have opposite signals, i.e.:

a) When the deflection is prescribed on the whole boundary (like a simply supported plate from all sides): The effect of GNL is computed from integrations performed only on sides of cells inside the domain but not on sides on the boundary of the plate;

b) When the deflection is not prescribed on the boundary portion of the plate $\left(\Gamma_{\mathrm{f}}\right)$ : The effect of GNL is computed from integrations performed on sides of cells inside the domain and on sides of cells on the boundary portion $\Gamma_{\mathrm{f}}$.

The basic inverse iteration and the Rayleigh quotient were used to perform the eigenvalue analysis. [23], i.e.:

$$
A x^{(k+1)}=x^{k}
$$




$$
\lambda_{k}=\frac{\left(x^{(k+1)}, x^{k}\right)}{\left(x^{(k+1)}, x^{(k+1)}\right)}
$$

The basic inverse iteration procedure is very efficient to compute the lowest eigenvalue with corresponding eigenvector [23]. Equation (12) was not used explicitly, but starting with an eigenvector $\mathrm{x}^{1}$ with all elements equal to 1.0, values for displacements and tractions at nodes of boundary elements were found. These values were introduced in the discretized form of equation (11), i.e. the equation written in terms of matrices, to obtain the eigenvector $x^{2}$ and the lowest eigenvalue at the first iteration step was obtained by using equation (13). The iteration procedure continued until the absolute difference between values of successive eigenvalues was less than $10^{-5}$. The proof of the convergence for the lowest eigenvalue can be found in [23].

The Young modulus (E) was $206.9 \mathrm{GPa}$, the Poisson ration (v) was 0.3 and the shear parameter was $\pi^{2} / 12$ (Mindin). The buckling parameter $\mathrm{k}$ is obtained according to the boundary conditions: $\mathrm{S}=$ simply supported edge, $\mathrm{C}=$ clamped edge and $\mathrm{F}=$ free edge.

$$
k=\frac{a^{2} N_{c r}}{\pi^{2} D}
$$

The critical in-plane load is $\mathrm{N}_{\mathrm{cr}}$ and the length of the plate side is a.

Table 1: $\quad$ Buckling parameter $(\mathrm{k})$ of the first critical in-plane load.

\begin{tabular}{|c|c|c|c|c|r|}
\hline Type & $\mathrm{h} / \mathrm{L}$ & {$[24]$} & {$[25]$} & 64 cells & 100 cells \\
\hline SSSS & 0.001 & 4.0000 & 4.0478 & 4.0503 & 4.0325 \\
\hline & 0.050 & 3.9280 & 3.9879 & 3.9928 & 3.9753 \\
\hline & 0.100 & 3.7290 & 3.8227 & 3.8291 & 3.8129 \\
\hline & 0.200 & 3.1190 & 3.2850 & 3.2887 & 3.2770 \\
\hline SSSC & 0.001 & 4.8470 & 4.9235 & 4.9392 & 4.9069 \\
\hline & 0.050 & 4.7170 & 4.8161 & 4.8343 & 4.8028 \\
\hline & 0.100 & 4.3720 & 4.5248 & 4.5447 & 4.5166 \\
\hline & 0.200 & 3.4180 & 3.6467 & 3.6640 & 3.6452 \\
\hline CSSS & 0.001 & 5.7400 & 5.8310 & 5.8167 & 5.7897 \\
\hline & 0.050 & 5.5740 & 5.6878 & 5.6710 & 5.6451 \\
\hline & 0.100 & 5.1400 & 5.3116 & 5.2813 & 5.2585 \\
\hline & 0.200 & 3.8760 & 4.1930 & 4.1852 & 4.1711 \\
\hline SCSC & 0.001 & 6.7430 & 6.8910 & 6.9490 & 6.8776 \\
\hline & 0.050 & 6.4620 & 6.6597 & 6.7181 & 6.6506 \\
\hline & 0.100 & 5.7650 & 6.0566 & 6.1133 & 6.0559 \\
\hline & 0.200 & 4.1090 & 4.4540 & 4.4977 & 4.4637 \\
\hline CSCS & 0.001 & 7.6910 & 7.9673 & 7.9306 & 7.8484 \\
\hline & 0.050 & 7.2280 & 7.5346 & 7.5166 & 7.4416 \\
\hline & 0.100 & 6.1780 & 6.5342 & 6.5381 & 6.4800 \\
\hline & 0.200 & 4.0560 & 4.4696 & 4.3990 & 4.3718 \\
\hline & & & & &
\end{tabular}


The Spline Strip Method was used in [24] and the boundary element method in [25]. The term related to the effect of GNL was simplified in [25] with equilibrium equations for in-plane forces $\left(\mathrm{N}_{\alpha \beta, \alpha}=0\right)$ and second derivatives of the deflection appeared as result from the simplification. The meshes used in [25] were: 40 isoparametric linear boundary elements with 100 constant cells for results in Table 1 and 80 isoparametric linear boundary elements with 400 constant cells for Table 2. Results in Tables 1 and 2 were obtained with: 64 quadratic boundary elements (132 nodes) with 64 constant cells and 80 quadratic boundary elements (164 nodes) with 100 constant cells

Table 2: $\quad$ Buckling parameter (k) of the first critical load.

\begin{tabular}{|c|c|c|c|c|}
\hline Type & $\mathrm{h} / \mathrm{L}$ & {$[24]$} & {$[25]$} & 64 cells \\
\hline FSSS & 0.001 & 1.4020 & & 1.4135 \\
\hline & 0.010 & 1.4000 & & 1.4137 \\
\hline & 0.050 & 1.3780 & 1.3336 & 1.3958 \\
\hline & 0.100 & 1.3270 & 1.3003 & 1.3546 \\
\hline & 0.200 & 1.1730 & 1.1785 & 1.2260 \\
\hline FSCS & 0.001 & 1.6520 & & 1.6685 \\
\hline & 0.010 & 1.6500 & & 1.6681 \\
\hline & 0.050 & 1.6200 & 1.5628 & 1.6383 \\
\hline & 0.100 & 1.5560 & 1.5074 & 1.5739 \\
\hline & 0.200 & 1.3700 & 1.3321 & 1.3852 \\
\hline FSFS & 0.001 & 0.9523 & & 0.9586 \\
\hline & 0.010 & 0.9516 & & 0.9586 \\
\hline & 0.050 & 0.9412 & 0.9007 & 0.9499 \\
\hline & 0.100 & 0.9146 & 0.8836 & 0.9287 \\
\hline & 0.200 & 0.8274 & 0.8151 & 0.8562 \\
\hline
\end{tabular}

Table 3: Buckling parameter (k) of the first critical load for a simply supported SSSS plate.

\begin{tabular}{|c|c|c|c|c|c|}
\hline BE & Cells & {$[11]$} & Obtained & DRM & Points \\
\hline 20 & $5 \times 5$ & 4.241 & 4.122 & 4.189 & 25 \\
\hline 24 & $6 \times 6$ & 4.173 & 4.085 & 4.141 & 36 \\
\hline 28 & $7 \times 7$ & 4.143 & 4.063 & 4.060 & 49 \\
\hline 32 & $8 \times 8$ & 4.079 & 4.048 & 4.032 & 64 \\
\hline 36 & $9 \times 9$ & 4.068 & 4.038 & 3.985 & 81 \\
\hline 40 & $10 \times 10$ & 4.041 & 4.030 & 3.999 & 100 \\
\hline
\end{tabular}

Tables 1 and 2 show the results obtained with the present formulation were better than those obtained with the use of second derivatives of the deflection to introduce the effect of GNL [25] and close to those obtained by Mizusawa [24]. 
Furthermore, the numbers of cells were lower using the present formulation than those used in [25].

Table 3 shows a comparison between results obtained with the present formulation and those presented by Purbolaksono and Aliabadi [11]. There were used constant rectangular cells to discretize the domain and the Dual Reciprocity Method in [11]. The expected value for $\mathrm{k}$ was 4.000 according to [11] but no information was included on values for thickness or mechanical constants $(E, v)$ or length of the side of the plate. The number of boundary elements $(\mathrm{BE})$ and the number of cells (Cells) used in [11] were adopted to check results with the present formulation. The numbers of points used for the DRM in [11] were the same used for cells and they were not repeated in Table 3. Instability on values for the DRM can be noted when 81 and 100 points were used when the results were lower than the expected value.

\section{Conclusions}

Results obtained with the use of first derivatives for the deflection to introduce the effect of GNL were apparently better than those obtained with second derivatives of the deflection. In spite of the reduction on the effort for the numerical implementation (use of first derivative, only), the number of domain cells was reduced and the convergence was monotonic according to the increase of the number of cells.

\section{Acknowledgements}

The authors are grateful to CNPq and FAPESP (grant \#011/21500-6, Sao Paulo Research Foundation) for supporting the development of the research on plates.

\section{References}

[1] Reissner, E., The Effect of Transverse Shear Deformation on the Bending of Elastic Plates, Journal of Applied Mechanics, 1945.

[2] Mindlin, R.D., Influence of rotatory inertia and shear on flexural motions of isotropic elastic plates, Journal of Applied Mechanics, 1951.

[3] Timoshenko, S. P., Woinowsky-Krieger, S., Theory of Plates and Shells, McGraw-Hill Book Company, New York, 2nd Ed., 1959

[4] Jones N. Structural impact. Cambridge, MA: Cambridge University Press; 1989.

[5] Dawe, D.J., Roufaeil, O.L., Buckling of rectangular Mindlin plates, Comp. \& Str., v.15, n.4, pp. 461-471, 1982.

[6] Herrmann, G. and Armenakas, A.E., Vibration and stability of plates under initial stress. Trans. Am. Soc. Civil Engrs., 127, pp. 458-487, 1962.

[7] Doong, J.-L., Vibration and stability of an initially stressed thick plate according to a high-order deformation theory, J. Sound Vibr. 113, pp. $425-440$. 
[8] Matsunaga, H., Free vibration and stability of thick elastic plates subjected to in-plane forces, Int. J. Sol. \& Str., v. 31, n. 22, pp. 3113-3124, 1994.

[9] Kitipornchai, S., Xiang, Y., Wang, C.M., Liew, K.M., Buckling of Thick Skew Plates, Int. J. Num. Met. Engng, v. 36, pp. 1299-1310, 1993.

[10] Lei, X.Y., Huang, M.K., Wang, X.X., Geometrically nonlinear analysis of a Reissner's type plate by boundary element method, Comput. Struct. 37 (6), pp. 911-916, 1990.

[11] Purbolaksono, J., Aliabadi, M.H., Large deformation of shear deformable plate by boundary element method. J. Eng. Math. 51, pp. 211-230, 2005.

[12] Palermo Jr., L., On the harmonic solution for plate bending considering effects of the shear deformation and the forces in the middle plane, In: Boundary Elements and other Mesh Reduction Methods XXXVII, WIT Press, 2014.

[13] Palermo Jr., L., Free vibration considering effects of the shear deformation and the geometric non linearity in the plate bending. In: $20^{\text {th }}$ International Conference on Computational Structures Technology, Naples, 2014.

[14] Palermo Jr., L., An alternative dynamic fundamental solution for plate bending including the shear deformation effect. In: Boundary Elements and other Mesh Reduction Methods XXXV, WIT Press, 2013.

[15] Birman, V., Plate Structures, Springer, 2011.

[16] Weeën F., Application of the boundary integral equation method to Reissner's plate model, Int. J. Numer. Methods Eng., 18: pp. 1-10, 1982.

[17] Elsgoltz, L., Ecuaciones Diferenciales y Cálculo Variacional, Editorial Mir, Moscu, 1977.

[18] Katsikadelis, J.T., The Boundary Element Method for Plate Analysis, Elsevier Academic Press, 2014.

[19] Timoshenko, S.P., Gere, J.M., Theory of Elastic Stability, 2 ${ }^{\text {nd }}$ Ed. Dover Publication, New York, 1961.

[20] Palermo Jr., L., The tangential differential operator applied to a stress boundary integral equation for plate bending including the shear deformation effect, Eng Anal Bound Elem, 36, pp. 1213-1225, 2012

[21] Aliabadi, M.H., The boundary element method, applications in solids and structures, vol.2, John Wiley \& Sons Ltd., 2002.

[22] Telles, J.C.F, A self-adaptive coordinate transformation for efficient numerical evaluation of general boundary element integrals, Int. J. Num. Meth. Eng., 24, pp. 959-973, 1987.

[23] Wilkinson, J.H., The Algebraic Eigenvalue Problem, Oxford University Press, William Clowes \& Sons, London, 1972.

[24] Mizusawa, T., Buckling of rectangular Mindlin plates with tapered thickness by the spline strip method, Int. J. Sol. Struct., v.30, n.12, pp. 1663-77, 1993.

[25] Sakanaka, S.H., Determinação de Frequências Naturais e Cargas Críticas em Placas, incluindo o Efeito da Deformação por Cortante, com o Método dos Elementos de Contorno, Master of Engineering Dissertation (in Portuguese), State University of Campinas, 2006. 